\title{
Search for genetic variants associated with cutaneous malignant melanoma in the Ashkenazi Jewish population
}

\author{
J C Y Loo, A D Paterson, A Hao, M Shennan, H Peretz, Y Sidi, D Hogg, E Yakobson
}

J Med Genet 2005;42:e30 (http://www.jmedgenet.com/cgi/content/full/42/5/e30). doi: 10.1136/jmg.2004.027300

C utaneous malignant melanoma (CMM; MIM \#155601) comprises about $1 \%$ of all cancer cases in European populations, but its mortality is over $2 \%$ because of the propensity of this tumour to metastasise. Surveillance of CMM-prone individuals and populations should decrease the morbidity and mortality associated with this disease.

In approximately $5-12 \%$ of all melanoma cases, there is a family history of the disease, defined as having one or more affected first degree relatives. ${ }^{1}$ Germline mutations of $C D K N 2 A$, located on chromosome 9p21, cosegregate with affected cases in an estimated $20 \%$ of melanoma families worldwide (reviewed by Goldstein ${ }^{2}$ ). CDKN2A encodes two different tumour suppressor proteins: the CDK4/6 cell cycle inhibitor $\mathrm{pl} 6^{\mathrm{INK} 4 \mathrm{~A}}$ from exons $1 \alpha, 2$, and 3; and the MDM2 inhibitor $\mathrm{p} 14^{\mathrm{ARF}}$ from exons $1 \beta, 2$, and 3 . The frequency of discovering a CDKN2A mutation within a family increases with the number of affected relatives, ranging from $\sim 15 \%$ in families with two affected members, to $20-40 \%$ in those with three, and $70 \%$ in those with four or more (unpublished data from Hogg's laboratory; Kefford and $\mathrm{Mann}^{1}$ ). In addition, mutations in the CDK4 gene $^{34}$ and polymorphisms in the $M C I R$ gene $^{5}$ have been implicated rarely in melanoma predisposition.

To date, mutations in the two major predisposition genes, CDKN2A and CDK4, account for melanoma susceptibility in less than half of all melanoma families, ${ }^{6}$ and the genetic bases of the disease in the remaining families have not been found. Gillanders et al recently provided strong evidence for another melanoma predisposition locus at chromosome $1 p 22 .{ }^{7}$ Current research in melanoma genetics is hindered by the allelic and locus heterogeneities, which render whole genome linkage analysis difficult. In addition, the sample sizes required to study the effects of weak or modifier alleles in a genetically heterogeneous population are large. ${ }^{8}$

To address the problem of both allelic and locus heterogeneities, we have focused on populations that have undergone genetic bottlenecks and are genetically isolated. We hypothesise that familial melanoma patients within such populations arose from a limited number of founders and thus display a restricted number of melanoma predisposition alleles. These alleles are in linkage disequilibrium with adjacent markers. By searching for melanoma associated alleles and genotypes, we can isolate the predisposition loci and subsequently determine the variant or variants responsible.

Ashkenazi Jews originated in the Near East, and subsequently migrated to eastern and central Europe. Throughout history, the Ashkenazi Jewish population has experienced numerous bottlenecks ${ }^{9}$ which led to highly altered allele frequencies relative to the diverse ancestral population. This genetic drift is evident from the high incidences of multiple genetic disorders, attributable to founder mutations, ${ }^{10}{ }^{11}$ and the population bottlenecks and the practice of endogamy have also decreased genetic variability. ${ }^{9}{ }^{12}$

\section{Key points}

- Mutations in CDKN2A and to a lesser extent, CDK4, account for the genetic susceptibility to cutaneous malignant melanoma in only a subset of familial cases. The remaining families may have melanoma associated variants in the non-coding regions of CDKN2A (on chromosome 9p21), or in other regions of the genome, possibly in the newly suggested melanoma susceptibility locus on chromosome $1 p 22$.

- The Ashkenazi Jewish population is genetically more isolated and less variable than the general population, so it was hypothesised that it would have a limited number of melanoma associated variants, and that they would exist in linkage disequilibrium with adjacent markers.

- The four exons of CDKN2A from the genomic DNA of 58 Ashkenazi Jewish melanoma patients were examined, but no mutations were uncovered. In addition, none of these patients bore the Arg24Cys and Arg24His mutations in CDK4 that are associated with melanoma.

- Analysis of the frequencies of two CDKN2A polymorphisms that were previously shown to be different between patients and controls was also done, but no significant differences were found between Ashkenazi Jewish cases and ethnically matched controls. Genotype analyses using microsatellite markers at 9 p21 and $1 p 22$ did not reveal any allelic or genotypic associations with melanoma between these loci.

- Further investigation is required to determine the locus responsible for melanoma susceptibility in the Ashkenazi Jewish population.

The incidence of melanoma in Ashkenazi Jews is relatively high (table 1), but no CDKN2A mutations have been found in any Ashkenazi Jewish families. ${ }^{13}$ This population is therefore considered to be a promising setting for the identification of novel melanoma susceptibility genes. ${ }^{6}$ In this report we present the results of our search for CDKN2A mutations and polymorphisms, as well as $9 p 21$ and $1 p 22$ alleles and genotypes that are associated with melanoma, in our collection of Ashkenazi Jewish melanoma patients.

\section{METHODS}

Recruitment of patients and controls

Fifty eight Ashkenazi Jewish patients with malignant melanoma (table 2; fig 1) were recruited to this study as

Abbreviations: $C M M$, cutaneous malignant melanoma 
Table 1 Age standardised rates of melanoma incidence in the year 2000

\begin{tabular}{lrr}
\hline Population/region & Male & Female \\
\hline Israel: Jewish* & 16.87 & 12.38 \\
Israel: non-Jewish* & 1.39 & 2.63 \\
Israel & 9.44 & 9.81 \\
Western Asia & 0.60 & 1.21 \\
Eastern Europe & 5.22 & 5.01 \\
Western Europe & 7.01 & 8.13 \\
Australia/New Zealand & 39.88 & 32.32 \\
North America & 12.74 & 9.26 \\
\hline
\end{tabular}

Sources: Globocan 2000: http://www-dep.iarc.fr/globocan/ globocan.html and *personal communication: M Barchana, Head, Israel National Cancer Registry.

described by Yakobson et al. ${ }^{13}$ Twenty of these reported a family history of the disease, defined as having at least one first degree relative affected by melanoma. All tumours were confirmed to be malignant melanoma by hospital pathologists. Genomic DNA was extracted from peripheral blood of all 58 patients.

The 86 control DNA samples were a kind gift from Dr Steve Narod (Toronto). They were obtained from individuals of Ashkenazi Jewish background who had no personal history of cancer, but may have had a family history of breast or ovarian cancer.

\section{Germline CDKN2A and CDK4 sequencing}

For all samples, we sequenced exons $1 \alpha$ and 2 of $C D K N 2 A$ as described by Liu et al, ${ }^{14}$ and exon 3 as described in Loo et al. ${ }^{15}$ We also amplified exon $1 \beta$ using primers pl4Elb-f and pl4Elb-r (table 3), and sequenced using the same primers. We amplified exon 3 of CDKN2A using primers pl6E3TMF and pl6E3TMR (table 3 ) and the following amplification profile: $95^{\circ} \mathrm{C}$ for three minutes; 38 cycles of $95^{\circ} \mathrm{C}$ for 21 seconds, $66^{\circ} \mathrm{C}$ for 16 seconds, $72^{\circ} \mathrm{C}$ for 51 seconds; and $72^{\circ} \mathrm{C}$ for 22 minutes.

To screen for the two known melanoma associated mutations in $C D K 4^{3}{ }^{4}$ in patient samples, we amplified exon 2 of the gene using primers CDK4E2f and CDK4E2r (table 3), and sequenced the polymerase chain reaction (PCR) products using the same primers.

\section{Genotype analysis at the $9 p 21$ and the $1 p 22$ chromosomal regions}

To determine whether the chromosomal locus $9 p 21$ and $1 p 22$ are associated with melanoma, we carried out genotype analysis to search for alleles shared as a result of founder effect. The microsatellite markers used were D9S1749, 974, $942,1748,1604$, and 171 for $9 p 21$; and D1S435, 2779, 236, 2664, and 2726 for $1 p 22$ (fig 2). We obtained primer sequences for all markers from the Genome Database (http://www.gdb.org/). We added the sequence GTTTCT to the $5^{\prime}$ of the forward primer of D9S1749 according to the principles of the "PIGtailing" technique. ${ }^{16}$ This increased the clarity of the genotypes by ensuring that non-templated adenylation by Taq polymerase occurs to $100 \%$ of labelled PCR products. We carried out PCR (annealing at $58^{\circ} \mathrm{C}$ ) and genotyping as described by Loo et al. ${ }^{15}$

\section{Statistical analyses}

For each marker, alleles were analysed if their frequencies were more than $10 \%$ in either patients or controls, and all other alleles were grouped together. We then compared the allele frequencies in the patients $v$ the controls for each marker. In examinations of genotypic associations, genotypes made up of alleles with frequencies of more than $10 \%$ in either patients or controls were analysed, and all other
Table 2 Descriptive statistics for patients and controls in the present study

\begin{tabular}{|c|c|c|}
\hline & Patients ( $n=58$ ) & Controls ( $n=86$ ) \\
\hline $\begin{array}{l}\text { Recruitment } \\
\text { Sex* } \\
\text { Age (years ) } \\
\text { (mean (SD)) } \\
\text { Ethnic background } \\
\text { Personal history of } \\
\text { melanoma } \\
\text { Multiple primary } \\
\text { melanomas } \\
\text { Family history of } \\
\text { melanoma }\end{array}$ & $\begin{array}{l}\text { Diagnosis: } 43.2 \text { (16.2) } \\
\text { Ashkenazi Jewish } \\
58 \text { patients }+(100 \%) \\
4 \text { patients (7\%) }\end{array}$ & $\begin{array}{l}\text { Dr S Narod, Toronto } \\
11 \text { male, } 75 \text { female } \\
\text { Recruitment: } 54.4 \text { (8.1) } \\
\text { Ashkenazi Jewish } \\
\text { None }\end{array}$ \\
\hline
\end{tabular}

*Sex distribution significantly different: $\chi^{2}=16.98, p=0.0000378$ $(\mathrm{df}=1)$.

†ncluding 10 patients whose samples have been analysed for CDKN2A mutations using single strand conformation polymorphism (SSCP). ${ }^{13}$

flncluding four individuals from two families.

genotypes were grouped together. We then compared the genotype frequencies in the patients $v$ the controls for each marker.

Genotype data were assessed for deviations from the Hardy-Weinberg Equilibrium separately for patient and control groups using the HWE program from the URL: http://linkage.rockefeller.edu/ott/linkutil.htm\#HWE. To test whether specific alleles or genotypes were associated with the disease, the genotype data were analysed using the $\chi^{2}$ test (EpiInfo6 from http://www.cdc.gov).

\section{RESULTS}

Table 2 presents the descriptive statistics for the patient and control groups in the present study. The statistically significant difference seen between the sex distributions in the patient and control groups is probably because the control group consisted primarily of individuals seeking genetic
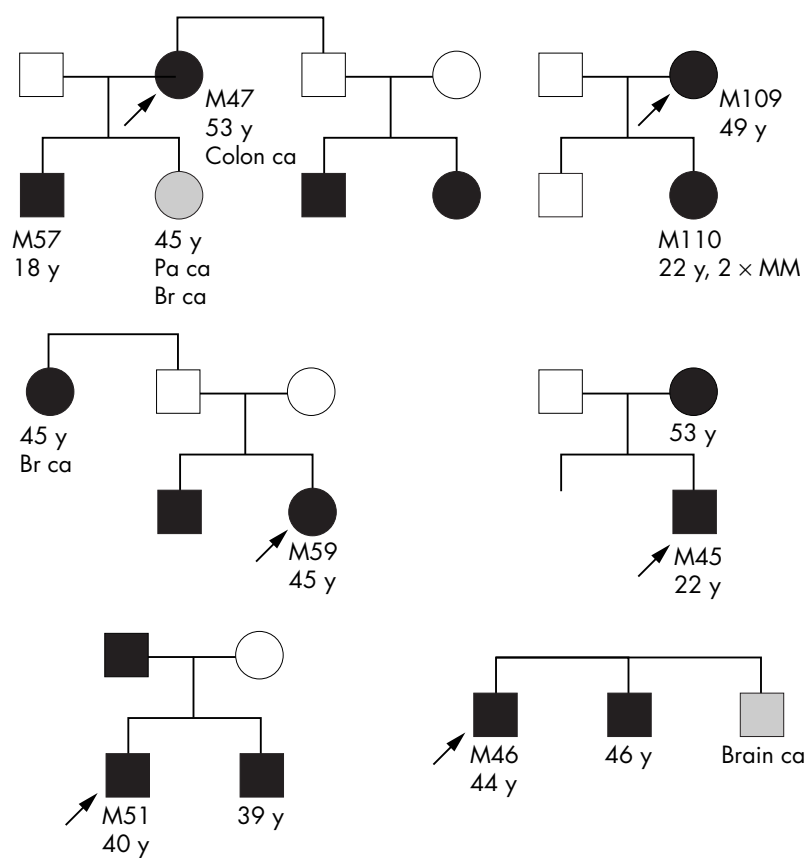

Figure 1 Examples of Ashkenazi Jewish melanoma pedigrees. Black symbols represent melanoma patients, and grey symbols represent individuals who suffer from cancers but not melanoma. Ages at diagnosis of the cancers are given under the symbols. Individuals in this study are designated by $M$ numbers. Additional information is available in table 2 . 
Table 3 New primers used in this study

\begin{tabular}{|c|c|c|c|c|}
\hline Primer & Sequence $\left(5^{\prime}\right.$ to $\left.3^{\prime}\right)$ & $\begin{array}{l}\text { Location of } 5^{\prime} \text { end } \\
\text { of primer }\end{array}$ & Direction & $\begin{array}{l}\text { Size of PCR } \\
\text { product (bp) }\end{array}$ \\
\hline $\begin{array}{l}\text { pl4E1b-f } \\
\text { pl4E1b-r } \\
\text { pl6E3TMF } \\
\text { pl6E3TMR } \\
\text { cdk4E2f } \\
\text { cdk4E2r }\end{array}$ & $\begin{array}{l}\text { CGTGTCAGGTGACGGATGTAGCTA } \\
\text { GGTACTGCGAGAACCACATGTCTAAG } \\
\text { GATGCGGGAAGGGAAAGGCCACATC } \\
\text { GACATTACGGTAGTGGGGGAAGGC } \\
\text { GGAGTCCCTITGTGCTGC } \\
\text { CCAGTCGCCTCAGTAAAGC }\end{array}$ & $\begin{array}{l}\text { c. }-359 \\
\text { IVS } 1+173 \\
\text { IVS } 2-380 \\
\text { c. } 658 \\
\text { IVS1-78 } \\
\text { c. } 190\end{array}$ & $\begin{array}{l}\text { Forward } \\
\text { Reverse } \\
\text { Forward } \\
\text { Reverse } \\
\text { Forward } \\
\text { Reverse }\end{array}$ & $\begin{array}{l}848 \\
581 \\
287\end{array}$ \\
\hline
\end{tabular}

bp, base pair; PCR, polymerase chain reaction.
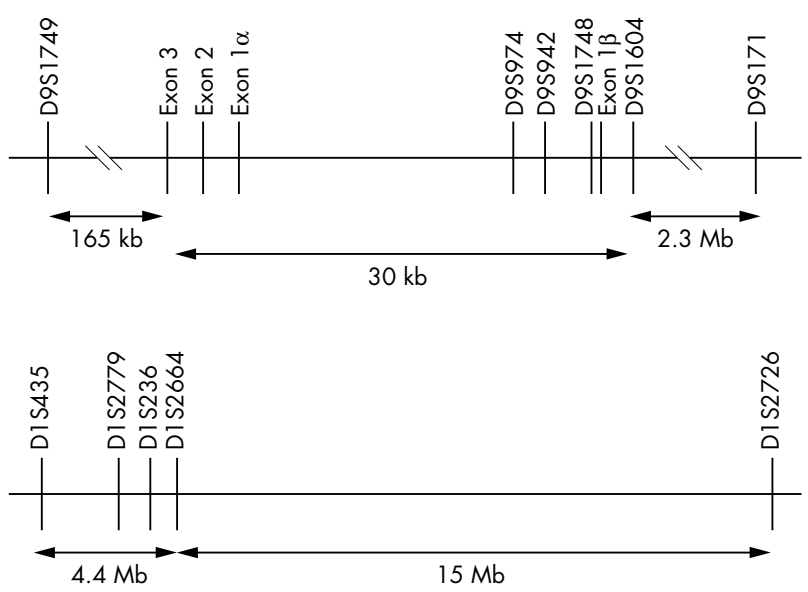

Figure 2 Schematic representations of the genetic markers used in the present study. Upper panel: microsatellite markers on chromosome 9p21 and the four exons of the CDKN2A gene. Lower panel: microsatellite markers on chromosome 1p22. Measurements are not to scale. Source: UCSC Genome Browser (May 2004): http:// genome.cse.ucsc.edu/index.html

testing for breast cancer susceptibility, a disease that predominantly affects women. As melanoma predisposition is not inherited in a sex restricted manner, the difference in sex distribution is unlikely to affect subsequent analyses. Twenty of the 58 patients (34\%, including four patients from two families) reported a family history, with clustering patterns consistent with an autosomal dominant mode of inheritance (fig 1). The remaining 66\% of the patients, though reported as sporadic cases, may still possess a genetic predisposition. Their "family history" of the disease may be unknown owing to dispersion of relatives, or is simply not observed if the penetrance is low and the family size is small.

We searched for melanoma associated mutations in the $C D K N 2 A$ and the CDK4 genes, but did not find any such variants in our patient population. We did not find a statistically significant difference (data not shown) between the frequencies of the C500G (rs11515) and C540T (rs3088440) polymorphisms, previously shown to be associated with melanoma. ${ }^{17} 18$

As the frequency of $C D K N 2 A$ coding mutations does not account for all $9 p 21$ linked families in other populations studied, we sought for a difference in allele and genotype frequencies between patients and controls, which may be indicative of candidate non-coding variants present in linkage disequilibrium with genetic markers in $9 p 21$. The allele and genotype distributions for all six markers (fig 2) did not deviate from the HWE in either the patient or the control groups. Table 4 shows $9 p 21$ allelic and genotypic frequency comparisons between patient and control groups, along with the calculated heterozygosities. Of the markers where the analyses were available, none showed allelic or genotypic associations with melanoma.

Allelic and genotypic associations at the $1 p 22$ locus Gillanders et $a l^{7}$ provided strong evidence that a novel melanoma susceptibility locus is present at $1 p 22$. We therefore searched for associations between 1p22 and melanoma cases in the Ashkenazi Jewish population. We undertook the same types of analyses for $1 p 22$ as we did for $9 p 21$, but found no significant allelic or genotypic associations with melanoma (table 5).

\section{DISCUSSION}

Multiple lines of evidence support a genetic basis for melanoma in Ashkenazi Jews. First, the incidence of cutaneous malignant melanoma in the Jewish population differs from that found in non-Jewish populations (table 1). Although differences in incidence rates among ethnic groups may reflect different environments and habits, they can also suggest that genetic backgrounds play a significant role in melanoma predisposition. Second, familial clustering suggests the segregation of mutant predisposition alleles. In our patient group, 20 of 58 patients (34\%; including four patients

Table 4 Association analyses for six microsatellite markers in 9p21

\begin{tabular}{|c|c|c|c|c|c|c|c|c|}
\hline \multirow[b]{2}{*}{ Marker } & \multicolumn{2}{|c|}{ Heterozygosities } & \multicolumn{3}{|c|}{ Allelic association } & \multicolumn{3}{|c|}{ Genotypic association } \\
\hline & Patients & Controls & $\chi^{2}$ & df & p value & $\chi^{2}$ & Df & p Value \\
\hline D9S1749 & 0.95 & 0.99 & 0.07 & 1 & 0.78 & \multicolumn{3}{|c|}{ Not analysed* } \\
\hline D9S974 & 0.95 & 0.74 & 0.51 & 3 & 0.51 & 14.9 & 6 & $0.021 \dagger$ \\
\hline D9S942 & 0.93 & 0.93 & 0.27 & 3 & 0.26 & 1.41 & 4 & 0.84 \\
\hline D9S1748 & 0.90 & 0.87 & 0.92 & 6 & 0.92 & \multicolumn{3}{|c|}{ Not analysed $\ddagger$} \\
\hline D9S1604 & 0.55 & 0.36 & 2.67 & 1 & 0.10 & 4.94 & 2 & 0.084 \\
\hline D9S171 & 0.75 & 0.75 & 3.26 & 3 & 0.35 & 7.10 & 5 & 0.21 \\
\hline
\end{tabular}

*The analysis for D9S1749 could not be carried out because there were no genotypes that were comprised of alleles that both had frequencies of $>10 \%$.

tWith the Bonferroni correction, the critical p value becomes $0.05 / 6=0.0083$. As 0.021 is $>0.0083$, the result is not statistically significant.

$\ddagger D 9 S 1748$ had too many genotypes that were comprised of alleles that both had frequencies of $>10 \%$; they therefore could not be analysed using the Epilnfo6 $\chi^{2}$ function. 
Table 5 Association analyses for six microsatellite markers in $1 p 22$

\begin{tabular}{|c|c|c|c|c|c|c|c|c|}
\hline \multirow[b]{2}{*}{ Marker } & \multicolumn{2}{|c|}{ Heterozygosities } & \multicolumn{3}{|c|}{ Allelic association } & \multicolumn{3}{|c|}{ Genotypic association } \\
\hline & Patients & Controls & $\chi^{2}$ & df & p value & $\chi^{2}$ & Df & $\mathrm{p}$ Value \\
\hline D1S435 & 0.75 & 0.70 & 5.78 & 3 & 0.123 & 3.60 & 6 & 0.730 \\
\hline D1S2779 & 0.70 & 0.76 & 1.65 & 2 & 0.437 & 1.76 & 3 & 0.625 \\
\hline D1S236 & 0.82 & 0.85 & 2.38 & 3 & 0.497 & 2.20 & 6 & 0.901 \\
\hline D1S2664 & 0.74 & 0.70 & 1.55 & 3 & 0.670 & 4.31 & 6 & 0.635 \\
\hline DIS2726 & 0.68 & 0.76 & 4.57 & 3 & 0.205 & 5.42 & 5 & 0.366 \\
\hline
\end{tabular}

from two families) reported a family history of the disease, with some having multiple affected relatives (fig 1). Third, some individuals-such as the four $(7 \%)$ in our series-are afflicted with multiple primary melanomas, or develop melanoma at an early age, observations that are also consistent with an underlying genetic predisposition. ${ }^{19}$ The early age of diagnosis of melanoma for some of the patients (for example, two patients diagnosed at age 22 in fig 1) possibly reflects accelerated tumour formation kinetics.

Despite the evidence pointing to the cosegregation of genetic variants with melanoma predisposition in the Ashkenazi Jewish population, no such variants have so far been uncovered. In an earlier study, Yakobson et $a l^{13}$ found two CDKN2A mutations, Glyl01Trp and Gly122Val, in two Israeli families, but neither is of Ashkenazi Jewish origin. Later, Yakobson et $a^{20}$ uncovered a Val59Gly mutation in four melanoma pedigrees. The concordance of the microsatellites at this chromosomal region shows that the mutation in these four families is probably derived from one Mediterranean non-Ashkenazi founder. The genetic basis for melanoma susceptibility in the Ashkenazi Jewish population thus remains unknown and is the focus of the present study.

Analyses of mtDNA haplogroups ${ }^{18}$ and the non-recombining regions of the $\mathrm{Y}$ chromosome ${ }^{12}$ have shown that a major genetic drift has occurred in the history of the Ashkenazi Jewish population. Our strategy of taking advantage of the linkage disequilibrium between markers that resulted from recent founder effects should have enabled us to define genetic associations with melanoma at the $9 p 21$ and $1 p 22$ candidate loci. However, we were unable to find any allelic or genotypic associations between either of the two single nucleotide polymorphisms (exon 3 of $C D K N 2 A$ ) or the 11 microsatellite markers (9p21 and 1p22) and melanoma. There are several possible reasons for our findings; for example, it is possible that even within the Ashkenazi Jewish population, numerous melanoma predisposition variants exist, and our sample size did not allow us to find a definitive association that is statistically significant; furthermore, the melanoma predisposition alleles for melanoma are not located in the $9 p 21$ or $1 p 22$ chromosomal regions for the Ashkenazi population. These two possibilities are not mutually exclusive.

Investigation by Catalin et al (Catalin M, personal communication) on 24 Ashkenazi Jewish families with malignant melanoma and neural system tumour syndrome (MMNST) has also found no predisposing mutations in $C D K N 2 A$. These results, together with ours ( 82 families in all) show that $C D K N 2 A$ is very unlikely to be the melanoma susceptibility locus for the Ashkenazi Jewish population. Further investigation should uncover a novel locus (or multiple loci) or environmental causes that underlie melanoma susceptibility of Ashkenazi Jews. The knowledge will allow the identification and surveillance of at-risk families, and thus decrease the morbidity and mortality associated with this disease.

\section{ACKNOWLEDGEMENTS}

We thank the individuals and families who participated in this study; Dr Steve Narod for control DNA samples; Dr Micha Barchana for information from the Israel National Cancer Registry; and members of the Hogg Lab for helpful discussions. JCYL is a recipient of an Ontario Graduate Scholarship in Science and Technology. ADP holds a Canada Research Chair in Genetics of Complex Diseases and is supported by Genome Canada. This work was supported by the National Cancer Institute of Canada (NCIC).

\section{Authors' affiliations}

J C Y Loo, Department of Medical Biophysics, University of Toronto, Toronto, Canada

A D Paterson, Program in Genetic and Genomic Biology, Hospital for Sick Children, Toronto

A Hao, M Shennan, Department of Clinical Sciences, University of Toronto

H Peretz, Laboratory of Clinical Biochemistry, Tel Aviv University, Sourasky Medical Centre, Tel Aviv, Israel

Y Sidi, E Yakobson, Molecular Cell Biology Laboratory, Department of Internal Medicine " $\mathrm{C}$ ", Sheba Medical Centre, Tel-Hashomer, Israel D Hogg, Department of Medicine, University of Toronto

Competing interests: none declared

Correspondence to: Dr David Hogg, University of Toronto, Medical Sciences Building, Room 7368, Toronto, Ontario, M5S 1A8 Canada; david.hogg@utoronto.ca

Received 17 September 2004

Revised version received 20 December 2004

Accepted for publication 21 December 2004

\section{REFERENCES}

1 Kefford RF, Mann GJ. Is there a role for genetic testing in patients with melanoma? Curr Opin Oncol 2003;15:157-61.

2 Goldstein AM. Familial melanoma, pancreatic cancer and germline CDKN2A mutations. Hum Mutat 2004;23:630.

3 Zuo L, Weger J, Yang Q, Goldstein AM, Tucker MA, Walker GJ, Hayward N, Dracopoli NC. Germline mutations in the p16INK4a binding domain of CDK4 in familial melanoma. Nat Genet 1996;12:97-9.

4 Soufir N, Avril MF, Chompret A, Demenais F, Bombled J, Spatz A, StoppaLyonnet D, Benard J, Bressac-de Paillerets B. Prevalence of p16 and CDK4 germline mutations in 48 melanoma-prone families in France. Hum Mol Genet 1998;7:209-16.

5 Sturm RA. Skin colour and skin cancer-MC1R, the genetic link. Melanoma Res 2002; 12:405-16.

6 Hayward NK. Genetics of melanoma predisposition. Oncogene 2003;22:3053-62.

7 Gillanders $\mathrm{E}$, Juo $\mathrm{SH}$, Holland EA, Jones $M$, Nancarrow D, Freas-Lutz D, Sood R, Park N, Faruque M, Markey C, Kefford RF, Palmer J, Bergman W, Bishop DT, Tucker MA, Bressac-de Paillerets B, Hansson J, Stark M, Gruis N, Bishop JN, Goldstein AM, Bailey-Wilson JE, Mann GJ, Hayward N, Trent J; Lund Melanoma Study Group; Melanoma Genetics Consortium. Localization of a novel melanoma susceptibility locus to $1 \mathrm{p} 22$. Am J Hum Genet 2003;73:301-13

8 Cordell HJ. Sample size requirements to control for stochastic variation in magnitude and location of allele-sharing linkage statistics in affected sibling pairs. Ann Hum Genet 2001;65:491-502.

9 Behar DM, Hammer MF, Garrigan D, Villems R, Bonne-Tamir B, Richards M, Gurwitz D, Rosengarten D, Kaplan M, Della Pergola S, Quintana-Murci L, Skorecki K. MtDNA evidence for a genetic bottleneck in the early history of the Ashkenazi Jewish population. Eur J Hum Genet 2004;12:355-64.

10 Neuhausen SL, Godwin AK, Gershoni-Baruch R, Schubert E, Garber J Stoppa-Lyonnet D, Olah E, Csokay B, Serova O, Lalloo F, Osorio A, Stratton M, Offit K, Boyd J, Caligo MA, Scott RJ, Schofield A, Teugels E, Schwab M, Cannon-Albright L, Bishop T, Easton D, Benitez J, King MC, 
Goldgar D, et al. Haplotype and phenotype analysis of nine recurrent BRCA2 mutations in 111 families: results of an international study. Am J Hum Genet 1998;62:1381-8.

11 Frisch A, Colombo R, Michaelovsky E, Karpati M, Goldman B, Peleg L. Origin and spread of the 1278insTATC mutation causing Tay-Sachs disease in Ashkenazi Jews: genetic drift as a robust and parsimonious hypothesis. Hum Genet 2004; 114:366-76.

12 Behar DM, Garrigan D, Kaplan ME, Mobasher Z, Rosengarten D, Karafet TM Quintana-Murci L, Ostrer H, Skorecki K, Hammer MF. Contrasting patterns of Y chromosome variation in Ashkenazi Jewish and host non-Jewish European populations. Hum Genet 2004; 114:354-65.

13 Yakobson E, Shemesh P, Azizi E, Winkler E, Lassam N, Hogg D, Brookes S Peters $G$, Lotem $M$, Zlotogorski A, Landau M, Safro M, Shafir R, Friedman $E$, Peretz $\mathrm{H}$. Two p16 (CDKN2A) germline mutations in 30 Israeli melanoma families. Eur J Hum Genet 2000;8:590-6.

14 Liu L, Dilworth D, Gao L, Monzon J, Summers A, Lassam N, Hogg D. Mutation of the CDKN2A 5' UTR creates an aberrant initiation codon and predisposes to melanoma. Nat Genet 1999;21:128-32.

15 Loo JC, Liu L, Hao A, Agatep R, Shennan M, Summers A, Goldstein AM, Tucker MA, Deters C, Fusaro R, Blazer K, Weitzel J, Lassam N, Lynch H,
Hogg D. Germline splicing mutations of CDKN2A predispose to melanoma. Oncogene 2003;22:6387-94.

16 Brownstein MJ, Carpten JD, Smith JR. Modulation of non-templated nucleotide addition by Taq DNA polymerase: primer modifications that facilitate genotyping. Biotechniques 1996;20:1004-6,1008-10.

17 Aitken J, Welch J, Duffy D, Milligan A, Green A, Martin N, Hayward N. CDKN2A variants in a population-based sample of Queensland families with melanoma. J Natl Cancer Inst 1999;91:446-52.

18 Kumar R, Smeds J, Berggren P, Straume O, Rozell BL, Akslen LA, Hemminki K. A single nucleotide polymorphism in the $3^{\prime}$ untranslated region of the CDKN2A gene is common in sporadic primary melanomas but mutations in the CDKN2B, CDKN2C, CDK4 and p53 genes are rare. Int J Cancer 2001;95:388-93.

19 Knudson AG. Mutation and cancer: statistical study of retinoblastoma. Proc Natl Acad Sci USA 1971;68:820-3.

20 Yakobson E, Eisenberg S, Isacson R, Halle D, Levy-Lahad E, Catane R, Safro M, Sobolev V, Huot T, Peters G, Ruiz A, Malvehy J, Puig S, Chompret A, Avril MF, Shafir R, Peretz H, Bressac-de Paillerets B. A single Mediterranean, possibly Jewish, origin for the Val59Gly CDKN2A mutation in four melanoma-prone families. Eur J Hum Genet 2003;11:288-96. 